\title{
Brugada Syndrome Inducted Ventricular Fibrillation Successfully Supported With Veno-arterial Extracorporeal Membrane Oxygenation and Continuous Renal Replacement Therapy:A Case Report
}

\section{Chengfeng Huang}

Jinan University First Affiliated Hospital

\section{Shifang Huang}

Jinan University First Affiliated Hospital

Jiawen Huang

Jinan University First Affiliated Hospital

Xiaoshen Zhang

Jinan University First Affiliated Hospital https://orcid.org/0000-0002-9984-8380

Fanna Liu ( $\nabla$ tliufana@jnu.edu.cn )

The First Affiliated Hospital ,Jinan University, Guangzhou

Hua Lu

Jinan University First Affiliated Hospital

Xiaohui Li

Jinan University First Affiliated Hospital

Yalan Li

Jinan University First Affiliated Hospital

\section{Case report}

Keywords: Brugada syndrome, Ventricular fibrillation, Veno-arterial extracorporeal membrane oxygenation, continuous renal replacement therapy, extracorporeal cardiopulmonary resuscitation

Posted Date: September 25th, 2020

DOI: https://doi.org/10.21203/rs.3.rs-77349/v1

License: (c) (1) This work is licensed under a Creative Commons Attribution 4.0 International License.

Read Full License 


\section{Abstract}

\section{Background}

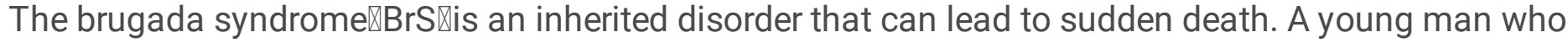
experienced sudden cardiac arrest caused by Brs was successfully rescued after 2hour cardiopulmonary resuscitation (CPR). This is a case that has raised concerns in China (more than 200 million people read this news via the internet).

Case presentation

A 28 years old young man was successfully managed with Veno-arterial ECMO (VA-ECMO) and continuous renal replacement therapy (CRRT) after more than 2 hours CPR. The diagnosis of BrS was identified during ECMO supported, and further ECG screening found two asymptomatic patients.

\section{Conclusion}

$\mathrm{BrS}$ can lead to malignant arrhythmia. VA-ECMO is the rescue treatment for patients with cardiac arrest who cannot be cured by conventional resuscitation. VA-ECMO combined with CRRT may help to improve the prognosis of critically ill patients.

\section{Specificity Of The Case}

It has been reported that the incident of BrS pattern on electrocardiogram (ECG) has ranged from $0.12 \%$ to $0.8 \%$. It has been considered responsible for $4 \%$ to $12 \%$ of all sudden deaths and up to $20 \%$ of sudden deaths in patients with structurally normal hearts ${ }^{[1]}$. In this case, prolonged CPR rescue was successful, and we believe that ECMO combined with CRRT improved the success rate of rescue.

\section{Background}

The brugada syndrome $\bigotimes \mathrm{B} r S \bigotimes$ is an inherited disorder that can lead to reccurent arrhythmic events or even sudden death. Even in asymptomatic patients, the malignant ventricular arrhythmia rate is up to $12 \%$ at 10-year follow-up ${ }^{[1]}$. Extracorporeal cardiopulmonary resuscitation (ECPR) is an important rescue method for patients with cardiac arrest, but prolonged CPR is often less successful. In this case, a young man who experienced sudden cardiac arrest caused by Brs was successfully rescued after 2hour cardiopulmonary resuscitation (CPR). This is a case that has raised concerns in China (more than 200 million people read this news via the internet).

\section{Case Presentations}

A 28 years old young man was found unconscious in hospital who was a family member of a patient. CPR was performed immediately when the patient was aware of sudden cardiac arrest, followed by electrical defibrillation, tracheal intubation, and epinephrine treatment for arrhythmia. The initial 
potassium after cardiac arrest was $5.3 \mathrm{mmol} / \mathrm{l}$, and the $\mathrm{PH}$ was 6.85 . The patient's hemodynamics remained unstable, with monomorphic and polymorphic ventricular tachycardia, and recurrent cardiac arrests. The rescue went on while waiting for agreement of ECMO treatment. Two hours later, he was emergently cannulated with $16 \mathrm{~F}$ in the right femoral artery and $24 \mathrm{~F}$ in the right femoral vein, and supported by ECMO with flow rates greater than 70 milliliter/ (kilogram× minute). Although his hemodynamic stabilized gradually and sinus rhythm appeared after ECMO running, the urine output declined and a large amount of pink frothy sputum poured out from the endotracheal tube. In order to identify the cause of cardiac arrest, we sent the patient with ECMO for computed X-ray tomography (CT) scan. CT scan revealed no signs of pulmonary embolism or cerebral hemorrhage, but bilateral diffuse infiltration distributed in the middle and lower lung segments. Echocardiography showed no abnormal blood flow.

In order to maintain an adequate ECMO flow, continuous infusion of fluid is required, while urine output declined and creatinine was $90 \mu \mathrm{mol} / \mathrm{l}$. The patient was diagnosed with cardiopulmonary failure, severe metabolic acidosis and volume overload. Initiate of CRRT was conductive to redress internal milieu disorder, and balance fluid management.

Hospital day 2: The recovery of the heart and extensive pulmonary effusion lead to differential hypoxia, which exists in VA-ECMO patients with compromised lungs. By strengthening ventilator support, cleaning respiratory tract, dehydrating through CRRT to reduce lung exudation, we effectively improved respiratory function and alleviated the hypoxia.

Hospital day 4『VA-ECMO was discontinued after improvement of cardiopulmonary function. The patient developed an ECG as shown in figure1 on day 8. After excluding other heart disease, the diagnosis was BrS which was characterized by an ECG pattern of right bundle-branch block and ST-segment elevation in the right precordial leads (V1-V3) ${ }^{[1]}$. Patient withdraw from CRRT on hospital day 13. Implantable cardioverter-defibrillator (ICD) was implanted two days later (figure 2).

The family members had been underwent genetic test. Mutation in CSRP3 c.161C>T was detected in patient and patient's father (figure 3).

\section{Discussion}

\section{Treatment of BrS}

We present an excellent outcome of a long persisting malignant arrhythmia (more than 2 hour) causing by $\mathrm{BrS}$. The BrS is an inherited disease associated with malignant arrhythmia and sudden cardiac death. Diagnosis is based on a characteristic ECG pattern. Implantable cardioverter defibrillator (ICD) is an effective therapy treating symptomatic patient ${ }^{[1]}$. However, risk statification of asymptomatic patients remain a challenge, therefore treatment strategies for asymptomatic patients are still controversial. But the rate of arrhythmic events in asymptomatic BrS patients lead to a $12 \%$ at 10 -year follow-up in population with the mean age of 40 years ${ }^{[2-4]}$. Management of BrS patients is not only limited to ICD or 
medications, but also includes education and lifestyle changes to prevent malignant arrhythmia. Enough attention should be paid to fever of BrS patient, because fever is a precipitating factor that could induce malignant arrhythmia ${ }^{[5]}$. In addition, the use of some contraindicated substance should be avoided.

\section{Genetic studies of BrS}

The diagnosis and treatment of $\mathrm{BrS}$ were not influence by genetic results, but genetic mutation may explain symptoms in patients and it is beneficial to screen out high-risk asymptomatic patient. Currently, more than 500 pathogenic variations have been association of $\mathrm{BrS}$, but genetic analysis was

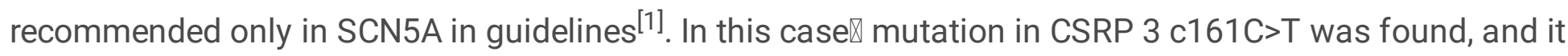
maybe a new potentially pathogenic variants associated with $\mathrm{BrS}$ although there is currently no relevant study.

\section{Therapy of refractory ventricular fibrillation[Combination of VA-ECMO and CRRT}

ECPR is an effective bridge therapy whilst the underlying cause of the cardiac arrest is determined ${ }^{[6]}$. However, the decision to initiate ECPR is always delayed until unsuccessful CPR. Ideally, ECMO should be initiated within $60 \mathrm{~min}$ after cardiac arrest, and patients with a low perfusion time $>90 \mathrm{~min}$ are less likely to benefit from ECMO ${ }^{[7]}$. However, high quality of chest compression is remain the most important measure influencing the efficacy of ECMO.

ECPR was be initiated after conventional resuscitation fail खthe low flow period always up to an hour before $\operatorname{ECPR}^{[6,7]}$. Acute kidney injury $(\mathrm{AKI})$ often occurs after cardiac arrest ${ }^{[8,9]}$. ECMO is also a high risk factor for AKI and fluid overload ${ }^{[10-12]}$. CRRT is an efficient therapy in the treatment of AKI and fluid overload. In this case, the CRRT was initiated in the early stage for improving fluid balance, enhancing the removal of inflammatory mediators and maintaining electrolyte balance. Early intervention through CRRT in ECPR patient may be beneficial to the reduction of the negative effects of fluid overload in ECMO ${ }^{[13]}$. According to previous research, most of the patients show recovery of renal function without continuation

of renal replacement before hospital discharged ${ }^{[14-17]}$, this finding could lead to a more active application of CRRT during ECMO.

ECPR patients are at risk of developing differential hypoxia, which may cause brain and heart hypoxia injury. In addition to adjusting ventilator parameters, CRRT may be beneficial to patient with acute respiratory distress syndrome through fluid restriction ${ }^{[18,19]}$. Fluid management presciently taken into account. CRRT can better facilitate fluid management strategy in the early stage of resuscitation. When respiratory function does not improve, a venoarteriovenous (VAV) ECMO should be considered, which adds VV blood flow into the pulmonary circulation of the existing VA-ECMO circuit. But VAV-ECMO is associated with recirculation, especially when the patients require a high VA-ECMO blood flow ${ }^{[20]}$, how to regulate the blood flow between double lumen cannula infusion lumen and femoral artery infusion according to the relative severity of heart versus lung failure is still a problem. 


\section{Conclusion}

BrS is related to the occurrence of malignant arrhythmia and should be taken seriously. Early detection and treatment can effectively prevent sudden cardiac death cause by this disease. Long low flow time may not benefit from VA-ECMO, but VA-ECMO is still the most effective therapy for the recovery organ perfusion after unsuccessful CPR (low flow state). Early combination of ECMO and CRRT may contribute to improve the outcome of critically ill patients.

\section{Abbreviations}

Brs: Brugada syndrome; CPR: Cardiopulmonary resuscitation; VA ECMO: Veno-arterial extracorporeal membrane oxygenation; CRRT: Continuous renal replacement therapy; CT: Computed X-ray tomography; ECG: Electrocardiogram; ICD: Implantable cardioverter defibrillator; ECPR: Extracorporeal cardiopulmonary resuscitation; AKI: Acute kidney injury; VAV-ECMO: Venoarteriovenous extracorporeal membrane oxygenation.

\section{Declarations}

\section{Ethics approval and consent to participate}

Not Applicable.

\section{Consent for publication}

The patient gave us written consent for publication of his details and images.

\section{Availability of data and materials}

Not Applicable.

\section{Competing interests}

The authors declare that they have no competing interests.

\section{Funding}

This study was supported by Guangdong Graduate Education Innovation Plan Project(2020XLLT10), 21st teaching reform projects of Jinan University(JG2019044). The finding sources had no role in study design, data collection and analysis, decision to publish, or preparation of the manuscript.

\section{Authors contributions}


CF $\mathrm{H}$ and FN L collected data and wrote the manuscript, SF H,CF H, H L, XH L, YL L took care of the patient, collected data, JW H wrote the manuscript, XS Z and FN L corrected the manuscript. All authors have read and approved the final version.

\section{Acknowledgment}

Not Applicable.

\section{References}

[1] Brugada J, Campuzano O, Arbelo E, et al. Present Status of Brugada Syndrome[J]. Journal of the American College of Cardiology. 2018, 72(9): 1046-1059.

[2] Sieira J, Ciconte G, Conte G, et al. Asymptomatic Brugada Syndrome: Clinical Characterization and Long Term Prognosis[J]. Circulation: Arrhythmia and Electrophysiology. 2015, 8(5): 1144-1150.

[3] Sieira J, Conte G, Ciconte G, et al. Clinical characterisation and long-term prognosis of women with Brugada syndrome[J]. Heart. 2016, 102(6): 452-458.

[4] Sieira J, Conte G, Ciconte G, et al. Prognostic Value of Programmed Electrical Stimulation in Brugada Syndrome邓20 Years Experience[J]. Circulation: Arrhythmia and Electrophysiology. 2015, 8(4): 777-784.

[5] Probst V, Denjoy I, Meregalli P G, et al. Clinical Aspects and Prognosis of Brugada Syndrome in Children[J]. Circulation. 2007, 115(15): 2042-2048.

[6] Hutin A, Abu-Habsa M, Burns B, et al. Early ECPR for out-of-hospital cardiac arrest_Best Practice in 2018[J]. Resuscitation. 2018, 130: 44-48.

[7] Wengenmayer T, Rombach S, Ramshorn F, et al. Influence of low-flow time on survival after extracorporeal cardiopulmonary resuscitation (eCPR囚[J]. Critical Care [Internet]. 2017, 21(1): 3-6.

[8] Geri G, Guillemet L, Dumas F, et al. Acute kidney injury after out-of-hospital cardiac arrest: risk factors and prognosis in a large cohort[J]. Intensive Care Medicine. 2015, 41(7): 1273-1280.

[9] Rundgren M, Ullén S, Morgan M P G, et al. Renal function after out-of-hospital cardiac arrest; the influence of temperature management and coronary angiography, a post hoc study of the target temperature management trial[J]. Critical Care. 2019, 23(1).

[10] Lin C, Chen Y, Tsai F, et al. RIFLE classification is predictive of short-term prognosis in critically ill patients with acute renal failure supported by extracorporeal membrane oxygenation[J]. Nephrology Dialysis Transplantation. 2006, 21(10): 2867-2873. 
[11] Smith A H, Hardison D C, Worden C R, et al. Acute renal failure during extracorporeal support in the pediatric cardiac patient[J]. ASAIO J. 2009, 55(4): 412-416.

[12] Yan X, Jia S, Meng X, et al. Acute kidney injury in adult postcardiotomy patients with extracorporeal membrane oxygenation: evaluation of the RIFLE classification and the Acute Kidney Injury Network criteria[J]. European Journal of Cardio-Thoracic Surgery. 2009.

[13] Schmidt M, Bailey M, Kelly J, et al. Impact of fluid balance on outcome of adult patients treated with extracorporeal membrane oxygenation[J]. Intensive Care Medicine. 2014, 40(9): 1256-1266.

[14] Cavagnaro F, Kattan J, Godoy L, et al. Continuous renal replacement therapy in neonates and young infants during extracorporeal membrane oxygenation[J]. The International journal of artificial organs. 2007, 30(3): 220.

[15] Hoover N G, Heard M, Reid C, et al. Enhanced fluid management with continuous venovenous hemofiltration in pediatric respiratory failure patients receiving extracorporeal membrane oxygenation support[J]. Intensive Care Medicine. 2008, 34(12): 2241-2247.

[16] Paden M L, Warshaw B L, Heard M L, et al. Recovery of renal function and survival after continuous renal replacement therapy during extracorporeal membrane oxygenation[J]. Pediatric Critical Care Medicine. 2011, 12(2): 153-158.

[17] Wolf M J, Chanani N K, Heard M L, et al. Early Renal Replacement Therapy During Pediatric Cardiac Extracorporeal Support Increases Mortality[J]. Ann Thorac Surg. 2013, 96: 917-922.

[18] Murphy C V, Schramm G E, Doherty J A, et al. The Importance of Fluid Management in Acute Lung Injury Secondary to Septic Shock[J]. Chest. 2009, 136(1): 102-109.

[19] Griffin B R, Liu K D, Teixeira J P. Critical Care Nephrology: Core Curriculum 2020[J]. American Journal of Kidney Diseases. 2020, 75(3): 435-452.

[20] Abrams D, Bacchetta M, Brodie D. Recirculation in Venovenous Extracorporeal Membrane Oxygenation[J]. ASAIO Journal. 2015, 61(2): 115-121.

\section{Figures}






Figure 1

ECG

CRRT 13D

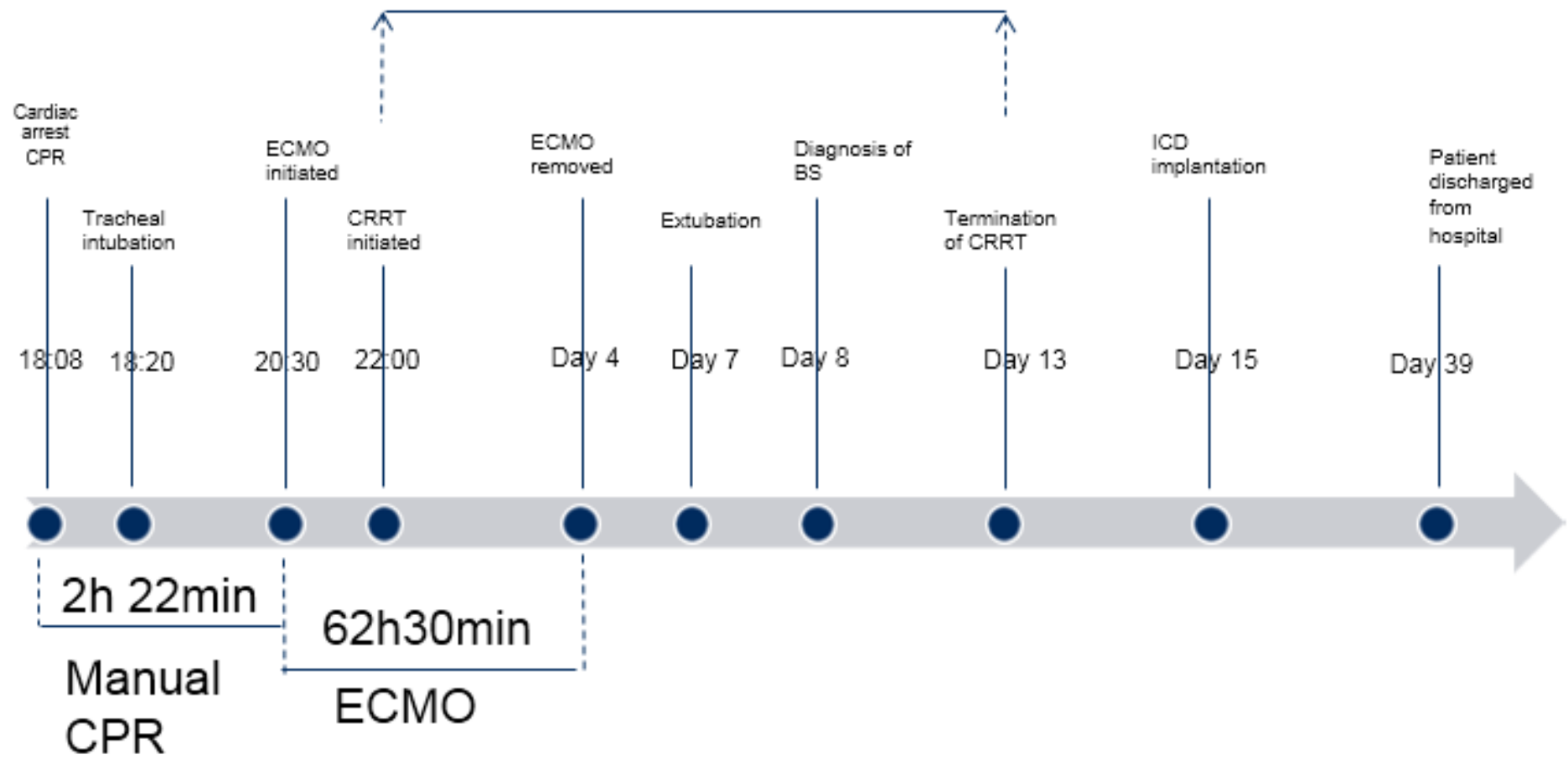

Figure 2 
Timeline of the rescue operation.

Patient CSRP3 c.161C $>\mathrm{T}$ Forward Heterozygous

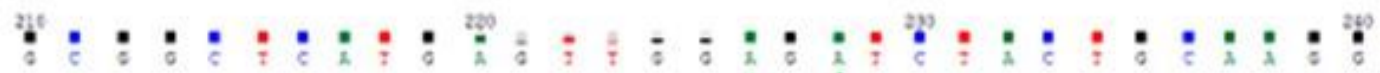

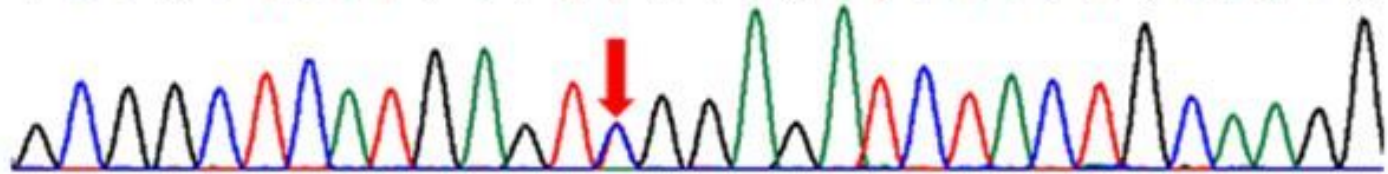

Patient's Father CSRP3 c.161C $>\mathrm{T}$ Forward Heterozygous

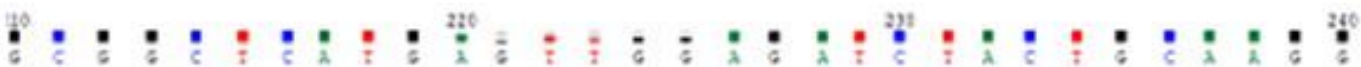

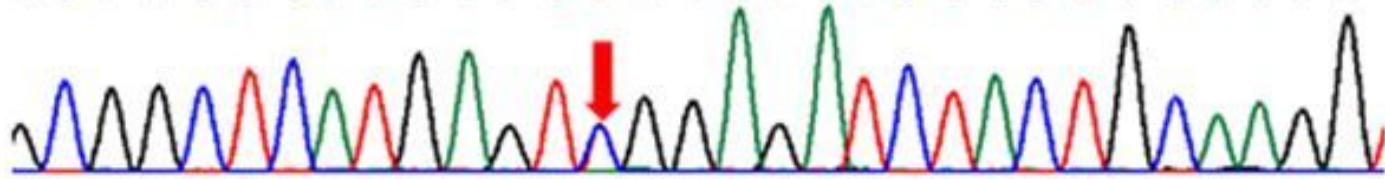

Patient's Mother $\quad$ CSRP3 c.161C $>$ T Forward Wild-type

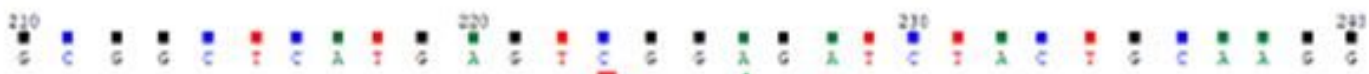

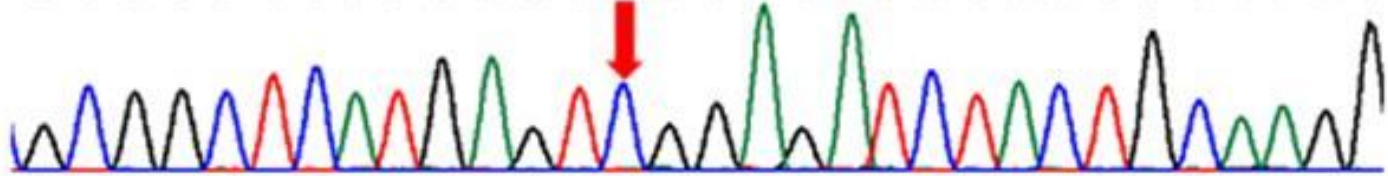

$\begin{array}{llll}\text { Patient's Sister A } & \text { CSRP3 } & \text { c.161C }>\text { T Forward } & \text { Wild-type }\end{array}$

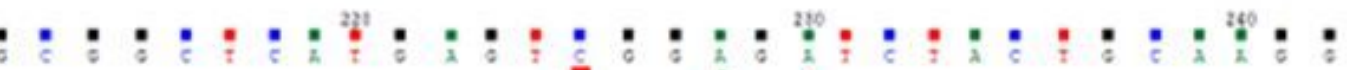

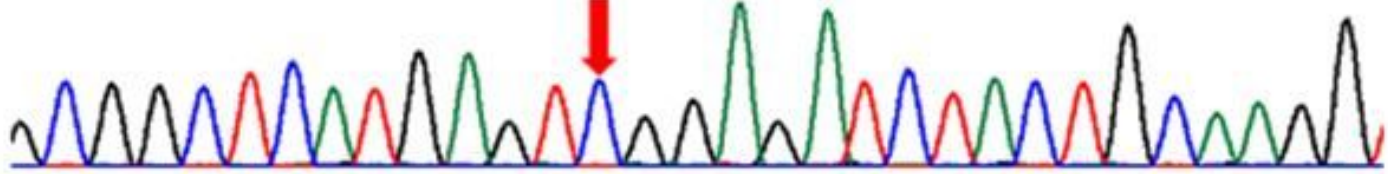

Patient's Sister B CSRP3 c.161C $>$ T Forward Wild-type

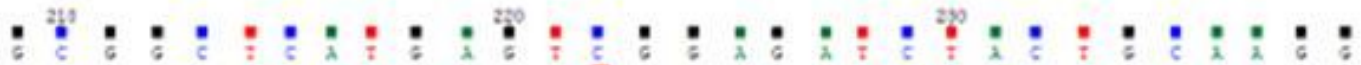

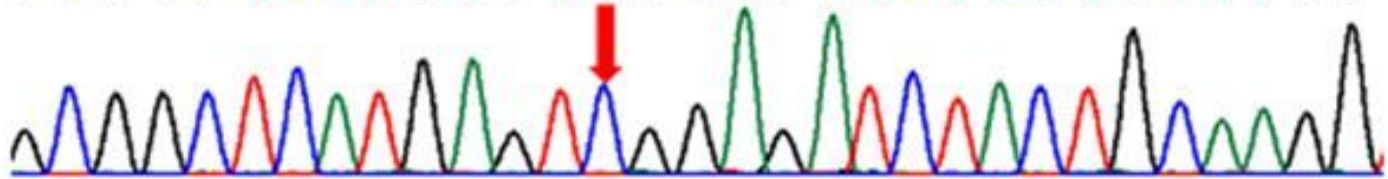

Figure 3

The nucleotide sequencing of CSRP3 c.161C>T polymorphism Supplementary Files 
This is a list of supplementary files associated with this preprint. Click to download.

- supplement1.jpg

- supplement2.jpg

- supplement3.jpg

- supplement4.jpg 